\title{
Ultrasound-guided paravertebral block for abdominal pain relief after transcatheter intrarterial chemoembolization of hepatic metastases
}

\author{
Anaya R., Baños V., Hoffmann R., Gil Sánchez JM., Italiano S., Moral MV. \\ Anaesthesiology Department. Hospital Santa Creu i Sant Pau (Barcelona). Spain.
}

\section{BACKGROUND}

- Transarterial chemoembolization with Irinotecan is an alternative to surgery in patients with liver metastases'. Up to 90\% of patients develop a Postembolization Syndrome (abdominal pain, nausea, vomiting and fever).

- Paravertebral block could minimize these symptoms. Previous reports showed the efficacy of this technique developed bilaterally and with multiple levels (T6-T8)2.

- We aimed to know whether one sole level and unilateral block is enough providing adequate pain relief during the transarterial chemoembolization with Irinotecan.

\section{CASE REPORT}

A 58 year old woman with colorrectal cancer history, treated with neoadjuvant irinotecan followed by laparoscopic right hemicolectomy, relapsed one year later with a solitary hepatic lesion (segment 4). She underwent two hepatic chemoembolization sessions.

For these procedures, we performed a single shot right side T8 paravertebral

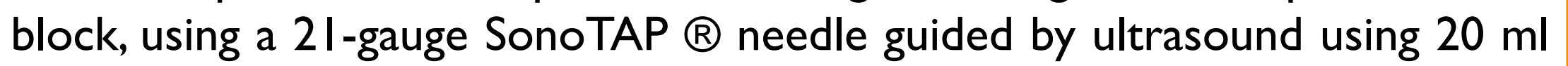
of ropivacaine 0 '75\%. Light sedation with propofol infusion was provided, as well as antiemetic profilaxis with $8 \mathrm{mg}$ dexametasone, I $\mathrm{mg}$ granisetrone and $40 \mathrm{mg}$ omeprazole.

We achieved a good analgesic control during the chemoembolization without any incidence. The Visual Analgesic Score was less than 4 during the following 24 hours and no opioids were required.
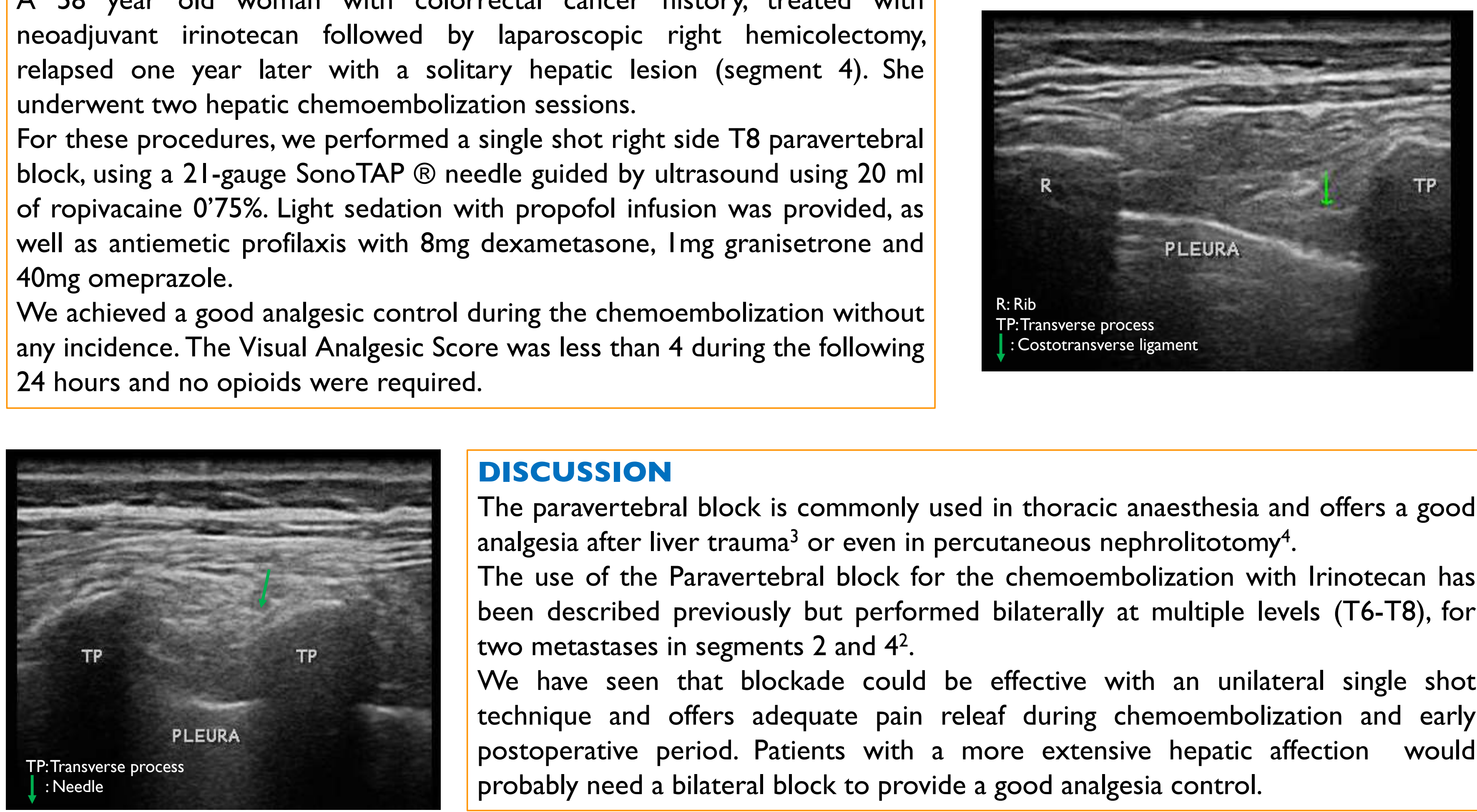

\section{DISCUSSION}

The paravertebral block is commonly used in thoracic anaesthesia and offers a good analgesia after liver trauma ${ }^{3}$ or even in percutaneous nephrolitotomy ${ }^{4}$.

The use of the Paravertebral block for the chemoembolization with Irinotecan has been described previously but performed bilaterally at multiple levels (T6-T8), for two metastases in segments 2 and $4^{2}$.

We have seen that blockade could be effective with an unilateral single shot technique and offers adequate pain releaf during chemoembolization and early postoperative period. Patients with a more extensive hepatic affection would probably need a bilateral block to provide a good analgesia control.

\section{LEARNING POINTS}

I. Hepatic chemoembolization with Irinotecan generates acute pain associated with important nausea and vomiting.

2. Single Shot Paravertebral block provides good analgesia with no opioid administration and subsequent lower nausea and vomiting and better patient comfort compared with general anaesthesia.

3. One sole level and unilateral block is enough to provide adequate pain relief in patients with a segment 4 hepatic lesion.

4. The use of ultrasound provides feasibility and better efficacy to the technique. 